\title{
Time-Series CCD Photometry of Northern Open Clusters Using the BOAO 1.8-m Telescope: III. NGC 2301
}

\author{
S.-L. Kim, B.-G. Park, M.-Y. Chun, H. Sung, Y.-B. Jeon, I.-S. Yuk, \\ Korea Astronomy Observatory, Korea \\ H. B. Ann, S. H. Lee
}

Department of Earth Science, Pusan National University, Korea

M. G. Lee

Department of Astronomy, Seoul National University, Korea

\begin{abstract}
A time-series CCD photometric survey of variable stars in open clusters has been in progress using $1.8-\mathrm{m}$ telescope at the Bohyunsan Optical Astronomy Observatory (BOAO) in Korea. We present preliminary results of time series photometry for the intermediate-age open cluster NGC 2301.
\end{abstract}

We have performed a long-term project on $U B V I$ CCD photometry and time series observation of open clusters. Its primary goals are to make a catalog of $U B V I$ photometry for open clusters which were little-studied before, and to search for variable stars in open clusters, particularly short-period (less than a few days) pulsating stars such as $\delta$ Sct stars, $\gamma$ Dor stars and SPBs which are recognized as important objects in studying stellar structure and testing evolution theory of an intermediate-mass $\left(1.3-7.0 M_{\odot}\right)$ main sequence star. More detailed descriptions on the project were given by Ann et al. (1999).

Time-series CCD observations of NGC 2301 were made by $1.8-\mathrm{m}$ telescope at BOAO. We have obtained a total of $212 \mathrm{~V}$ frames over six nights from 1999 February 6 to 13 with the SITe $2048 \times 2048$ CCD. We processed CCD images using the IRAF/CCDRED package. Instrumental magnitudes were obtained via the PSF (Point Spread Function) photometric routine. We applied the ensemble normalization technique (Gilliland \& Brown 1988) to standardize instrumental magnitudes of the time-series CCD frames.

In order to search for variable stars, we examined light variations of 923 stars among 944 stars in the observed field. The saturated stars brighter than $10^{\mathrm{m}}$ and the stars located within 20 pixels from the edge of the time-series CCD frames were excluded. Nine variable stars have been discovered (Table 1 and Fig. 1).

\section{References}

Ann, H. B., Lee, M. G., Chun, M.-Y., Kim, S.-L., et al. 1999, JKAS, 32, 7

Gilliland, R. L. \& Brown, T. M. 1988, PASP, 100, 754 


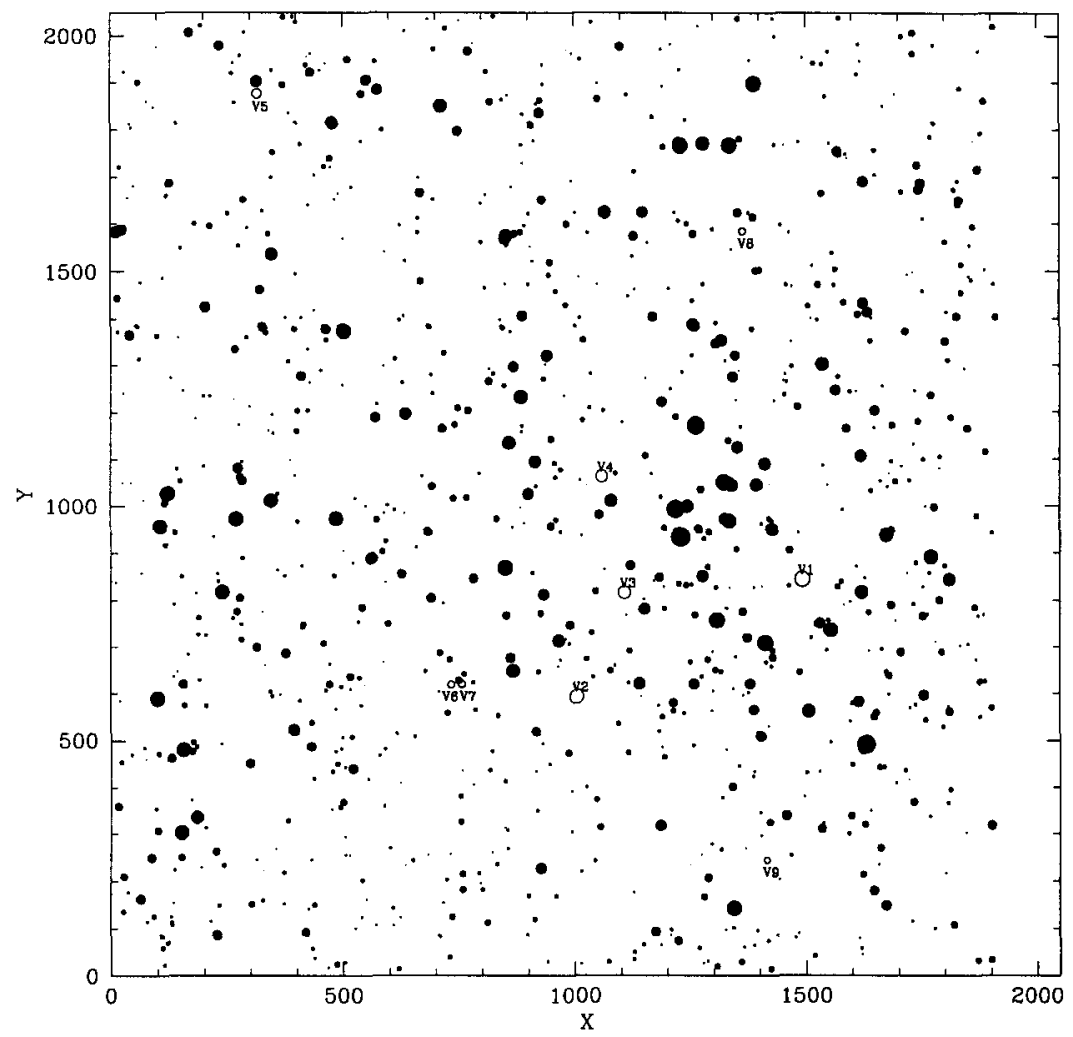

Figure 1. Observed CCD field $\left(11^{\prime} 6 \times 11^{\prime} \cdot 6\right)$ of NGC 2301 . Nine variable stars discovered in this study are marked by open circles. North is up and east is to the left.

Table 1. Nine variable stars discovered in NGC 2301.

\begin{tabular}{cccccccc}
\hline ID & $V$ & $B-V$ & Period & $\Delta V$ & Epoch $^{\dagger}$ & Type & $\mathrm{P}_{\mu}^{\ddagger}$ \\
\hline V1 & $10^{\mathrm{m}} 864$ & $0^{\mathrm{m}} \mathbf{m} 095$ & $0^{\mathrm{d}} 332$ & $\sim 0^{\mathrm{m}} 04$ & 2451216.97 & Field SPB & 0.00 \\
V2 & $11^{\mathrm{m}} 621$ & $0^{\mathrm{m}} 341$ & $0^{\mathrm{d}} 352(?)$ & $\sim 0^{\mathrm{m}} 03$ & 2451216.17 & $\gamma$ Dor & 0.91 \\
V3 & $12^{\mathrm{m}} 562$ & $0^{\mathrm{m}} \cdot 387$ & $0^{\mathrm{d}} 299$ & $\sim 0^{\mathrm{m}} 02$ & 2451216.06 & $\gamma$ Dor & 0.96 \\
V4 & $12^{\mathrm{m}} 708$ & $0^{\mathrm{m}} 368$ & $0^{\mathrm{d}} 340$ & $\sim 0^{\mathrm{m}} 04$ & 2451216.02 & $\gamma$ Dor & 0.93 \\
V5 & $14^{\mathrm{m}} 452$ & $0^{\mathrm{m}} 922$ & $0^{\mathrm{d}} \cdot 778$ & $\sim 0^{\mathrm{m}} 08$ & 2451216.01 & Eclipsing & - \\
V6 & $15^{\mathrm{m}} 872$ & $0^{\mathrm{m}} 925$ & $1^{\mathrm{d}} 102$ & $\sim 0^{\mathrm{m}} 15$ & 2451216.95 & Eclipsing & - \\
V7 & $15^{\mathrm{m}} 975$ & $0^{\mathrm{m}} 910$ & $0^{\mathrm{d}} 642$ & $\sim 0^{\mathrm{m}} 17$ & 2451216.18 & Eclipsing & - \\
V8 & $16^{\mathrm{m}} 075$ & $1^{\mathrm{m}} 097$ & $0^{\mathrm{d}} 250$ & $\sim 0^{\mathrm{m}} 09$ & 2451216.09 & Eclipsing & - \\
V9 & $16^{\mathrm{m}} 605$ & $1^{\mathrm{m}} 041$ & $0^{\mathrm{d}} 873$ & $\sim 0^{\mathrm{m}} 12$ & 2451216.17 & Eclipsing & - \\
\hline
\end{tabular}

$\dagger^{\dagger}$ : maximum brightness for pulsating stars and minimum for eclipsing binaries.

$\ddagger$ : open cluster database (obswww. unige.ch/webda) by J.-C. Mermilliod. 